\title{
Standardization Study of Internet Addiction Improvement Motivation Scale
}

\author{
Jae Woo Park ${ }^{1}$, Kee Hwan Park' ${ }^{1}$ In Jae Lee ${ }^{2}$, Min Kwon ${ }^{3}$ and Dai Jin Kim ${ }^{3 凶}$ \\ 'Department of Psychology, The Catholic University of Korea College of Social Science, Bucheon, Republic of Korea \\ ${ }^{2}$ Department of Social Rehabilitation, Hanshin University College of Human Service, Osan, Republic of Korea \\ ${ }^{3}$ Department of Psychiatry, Seoul St. Mary's Hospital, The Catholic University of Korea College of Medicine, Seoul, Republic of Korea
}

\begin{abstract}
Objective The purpose of this study was to develop a scale to measure motivation to improve Internet addiction. Motivation is known to be important to treat Internet addiction successfully. The reliability of the scale was assessed, and its concurrent validity was evaluated. Methods Ninety-two adolescents participated in this study. The basic demographic characteristics were recorded and the Korean version of the Stages of Readiness for Change and Eagerness for Treatment Scale for Internet Addiction (K-SOCRATES-I) was administered. Subsequently, the Internet Addiction Improvement Motivation Scale was developed using 10 questions based on the theory of motivation enhancement therapy and its precursor version designed for smoking cessation.

Results The motivation scale was composed of three subscales through factor analysis; each subscale had an adequate degree of reliability. In addition, the motivation scale had a high degree of validity based on its significant correlation with the K-SOCRATES-I. A cut-off score, which can be used to screen out individuals with low motivation, was suggested.

Conclusion The Internet Addiction Improvement Motivation Scale, composed of 10 questions developed in this study, was deemed a highly reliable and valid scale to measure a respondent's motivation to be treated for Internet addiction.
\end{abstract}

Psychiatry Investig 2012;9:373-378

Key Words Internet addiction, Motivation scale, Motivation enhancement therapy, Validation, Standardization.

\section{INTRODUCTION}

\section{Internet addiction worldwide}

The problem of Internet addiction has drawn the attention of researchers worldwide, and because the Internet industry continues to grow, the incident rate of the disorder is increasing. In the Netherlands, it has been reported that the incident rate of Internet addiction reaches as high as 1.5 to $3.0 \%$, and those who have Internet addiction have a difficult time adjusting to their school or workplace. ${ }^{1}$ According to another research study in Norway, $1 \%$ of the population can be classified as Internet addicted and $5.2 \%$ of the population can be classified as a latent risk group for Internet addiction. In particular,

Received: October 21, 2011 Revised: December 6, 2011

Accepted: March 19, 2012 Available online: November 15, 2012

$\triangle$ Correspondence: Dai Jin Kim, MD, PhD

Department of Psychiatry, Seoul St. Mary's Hospital, The Catholic University of Korea College of Medicine, 505 Banpo-dong, Seocho-gu, Seoul 137-040, Republic of Korea

Tel: +82-2-2258-6933, Fax: +82-2-594-3870, E-mail: kdj922@chollian.net

(a) This is an Open Access article distributed under the terms of the Creative Commons Attribution Non-Commercial License (http://creativecommons.org/licenses/bync/3.0) which permits unrestricted non-commercial use, distribution, and reproduction in any medium, provided the original work is properly cited. young male adults with a high education but low socioeconomic status are vulnerable to the disorder. ${ }^{2}$

In the case of Hong-Kong, $17 \%$ of research participants showed symptoms of Internet addiction and half experienced severe insomnia. ${ }^{3}$ With Internet addiction appearing to spread worldwide, it is becoming a disorder that aggravates many psychosocial problems.

Discussions of the concept and diagnosis criteria for Internet addiction are active in research circles. Goldberg used the term "addictive disorder" based on the substance addiction of Diagnostic and Statistical Manual for mental disorder 4th edition (DSM-IV) for the first time, and he refers to Internet addiction as "pathological computer use." Young also suggested Internet addiction diagnosis criteria, including obsessions with the Internet, tolerance, withdrawal symptoms, excessive computer use, lack of interest in other activities. He based these diagnostic criteria on those developed for pathological gambling. ${ }^{5}$

In this study, three criteria are adopted-tolerance, withdraw$\mathrm{al}$, and deterioration of functional level in everyday life-to conceptualize Internet addiction. 


\section{Internet addiction and measuring motivation for treatment in South Korea}

According to a study conducted in South Korea, Internet addiction was observed in more than $30 \%$ of people aged from 10 to more than 30 years old. In particular, $46.8 \%$ of those aged 10 to 19 years old showed signs of addiction. ${ }^{6}$ Another study reported that the prevalence of Internet addiction reached 9 to $40 \%$ among adolescent group in Korea. ${ }^{7}$ The prevalence rate of Internet addiction in South Korea is higher than any other country. Internet addiction, with such high prevalence, is associated with tolerance and withdrawal symptoms, much like other addictions. As such, more and more persons are exhibiting Internet addiction. Terminating the use of the Internet provokes various psychological symptoms, which eventually reduces the individual's functional level in daily life. It can thus be said that Internet addiction is a severe disorder. In this way, because the problem of Internet addiction in South Korea is more severe than other countries, our research focused on a Korean population.

Unlike other mental illnesses, addiction is strengthened through problem behaviors. Because of a low motivation to improve, the drop-out rate from treatment programs tends to be high. Actually, severe addiction can lead to the perception that the addictive behavior increases the motivation for treatment in some cases. ${ }^{8}$ Others, however, exhibit very low motivation even when participating in an active treatment situation. It is important, therefore, to identify high risk addicts early by evaluating and measuring the level to which they are motivated to improve and provide more intensive treatment for them.

\section{Previous studies and treatment for addiction in South Korea}

Whether dealing with addiction or other mental illness, what is most necessary is to develop scales to examine related concepts. To examine Internet addiction, an Internet addiction scale was developed by Young, which consisted of 20 questions. ${ }^{9}$ And in Korea, a K-scale, which considered Korean circumstances related with Internet addiction was developed by Kim et al. ${ }^{10}$ Such tools are useful for distinguishing persons with Internet addiction from normal users so that addicts can receive treatment. The scales are also important for identifying a latent risk group to provide education about how to prevent addiction. The K-scale classifies individuals beyond 2 and 1 standard deviation from a mean as either high or latent risk groups, respectively, with the total score and the scores of the subscales on tolerance, withdrawal, and disturbance of functioning as standards. ${ }^{10}$

In South Korea, the Ministry of Health and Welfare offers a voucher service to adolescents with Internet addiction. Th- rough the service, adolescents that meet the Ministry's family income criteria and are classified as high or latent risk groups with Internet addiction can be treated at nearly no cost. Because participants include both adolescents with a low motivation for treatment and those with a comparatively high motivation, we felt the need to screen for groups that need more intensive treatment at an earlier stage. To accomplish this, we developed the Internet Addiction Improvement Motivation Scale by modifying Kim's verified and standardized smoking cessation motivation scale (KSCMS), which was developed based on the motivational interview theory about smoking. Because Internet addiction is a behavioral addiction and nicotine addiction is a substance addiction, the scales that measure patterns of addiction cannot be interchangeable through simple modification. But a range of studies have suggested that the patients' motivation or intense desire to quit smoking represent a critical part of the process of smoking cessation. And motivational interview can be successfully applied to smoking cessation program. ${ }^{11}$ And that motivational interview can also be applied to internet addiction improvement program and according to research data, motivational interview program reduces internet using time and addiction level measured by K-scale significantly. ${ }^{8}$ Thus improving motivation and treatment of both behavioral addiction and substance addiction can be explained by the common theory of motivational interview and stage of change model. So questions from KSCMS can be adopted as questions for the Internet Addiction Improvement Motivation scale. KSCMS was developed to evaluate the motivation level that theoretically corresponds to the first three stages of the change model of motivational enhancement therapy. Author name pointed out however, that the third stage, preparation, has a problem. It is divided into two types of items that examine stages closer to preparation and to practice. The reliability, construct validity, and predictive validity of the KSCMS, however, were all found to be high. ${ }^{12}$

\section{Developing other addiction motivation scales}

The effect of motivational enhancement therapy has drawn significant attention or has been proven effective in treating various types of addiction, including dependence on alcohol. Motivation scales based on the theory of motivational enhancement therapy have also been developed. For alcohol dependence, a motivation scale called SOCRATES was developed, ${ }^{11,14}$ which classifies stages of change into pre-contemplation, contemplation, preparation, action, and maintenance based on the pattern of scores among subscales. K-SOCRATES was validated by adapting the scale in the South Korean context. ${ }^{14}$

For smoking cessation, we developed the K-SOCRATES-sm- 
oking by modifying the K-SOCRATES and proceeded with its validation. ${ }^{13}$ In addition, we developed and validated KSCMS, which consists of the first three of the five stages in the stage of a change model-pre-contemplation, contemplation, and preparation-to assess motivation for smoking cessation at the beginning of treatment. ${ }^{13}$ According to a preliminary study, however, although the total score of K-SOCRATES-Internet (K-SOCRATES-I) has high reliability and validity, its factor structure differs from the original version. Thus, it can be used as a total score, but cannot be used as sub-scale scores. Additional research is needed for full validation and standardization of K-SOCRATES-I. Furthermore, although K-SOCRATES-I is fully standardized successfully, Korean version of Internet Addiction Improvement Motivation Scale (KIAIMS) is more brief and easier to interpret than K-SOCRATES-I because the higher the score of every subscale of K-IAIMS, the higher the motivation for improvement.

In this study, we develop an Internet addiction version of the motivation scale based on KSCMS to screen for a highrisk group with low motivation to improve by evaluating the

Table 1. Demographic variables

\begin{tabular}{lc}
\hline \multicolumn{1}{c}{ Variable } & Content \\
\hline Age, $\mathrm{M}(\mathrm{SD})$ & $12.8(0.8)$ \\
Gender (Frequency) & Male (61)/Female (51) \\
General group (\%) & 84.0 \\
Latent addiction group (\%) & 9.6 \\
Addiction group (\%) & 4.4 \\
\hline
\end{tabular}

motivation level at the beginning of treatment. The scale is considered to be very helpful in differentiating between a group that can improve with standard interventions and a high-risk group that needs intensive intervention for improvement. We do this by assessing the motivation to improve addiction in the initial stages of treatment for Internet addiction.

\section{METHODS}

\section{Participants}

In total, 112 middle-school students who were subjects of the Internet addiction voucher service participated in this study. Their demographic characteristics are shown in Table 1. The subjects and their parents provided written informed consent after receiving a full explanation of the study purpose, procedures as approved by the Institutional Review Board of Seoul St. Mary’s Hospital.

\section{Procedure}

Ten questions to measure a client's motivation level to improve Internet addiction through treatment were posed based on the KSCMS. The goal was to screen for the poorly motivated group. This was done by assessing clients who need treatment early using 10 questions describing the characteristics of the first three stages of change in the motivational enhancement therapy. These questions were modified from 10 questions drawn from the KSCMS. The confirmed 10 questions were adapted by two specialists who were fluent in both English and Korean. Their English version is presented in Table 2.

Table 2. Factor structure, reliability and descriptive statistics of internet addiction improvement motivation scale

\begin{tabular}{|c|c|c|c|}
\hline \multirow{2}{*}{ Items } & \multicolumn{3}{|c|}{ Factor } \\
\hline & F1 & F2 & F3 \\
\hline 1 I contemplated effects of internet use on me. & & 0.531 & \\
\hline $\begin{array}{l}2 \text { I recognize that positive effects of appropriate internet use are much larger } \\
\text { than those of excess internet use. }\end{array}$ & & 0.660 & \\
\hline 3 I have many resources to succeed in reducing internet use. & & 0.782 & \\
\hline 4 I want to progress necessary stages for reducing internet use. & 0.556 & & \\
\hline 5 I let others know my attempt to control internet use. & 0.755 & & \\
\hline 6 I want to receive professional help to reduce internet use. & 0.615 & & \\
\hline 7 I think continuous internet use does not matter. & & & 0.336 \\
\hline 8 I did not contemplate my internet use problem. & & & 0.882 \\
\hline 9 I feel various discomforts from my internet use problem. & 0.641 & & \\
\hline 10 I do not want to reduce my internet use. & & & 0.595 \\
\hline Cronbach's alpha & 0.734 & 0.724 & 0.613 \\
\hline Total & & 0.733 & \\
\hline Variance explained (\%) & 16 & 22 & 18 \\
\hline Total & & 56 & \\
\hline
\end{tabular}

F1: preparation level motivation, F2: contemplation level motivation, F3: pre-contemplation level motivation 


\section{Measures}

The scale developed for this study included 10 questions specific to Internet addiction and related with the first three stages of change in motivational enhancement therapy: precontemplation, contemplation, and preparation. Each question was answered using a Likert scale anchored by $1=$ strongly disagree to $6=$ strongly agree. To avoid bias caused by random responses, some questions were reverse scored.

\section{K-Scale}

The K-scale was developed by Kim et al. to examine followings related to Internet use. It contains seven subscales-disturbance of functioning level, disturbance of reality testing, addictive automatic thought, withdrawal, virtual interpersonal relationships, deviant behavior, and tolerance. The addiction group and the latent addiction groups were classified with cut-off points either in the total score or the scores on the disturbance of functioning level, withdrawal, and tolerance measures, which account for the important portions in defining addiction. This study's internal consistency was 0.970 .

\section{K-SOCARTES-I}

To test the construct validity of the Internet Addiction Improvement Motivation Scale, we developed and administered the K-SOCRATES-I scale. The scale consists of three factors-recognition, ambivalence, and taking steps-through factor analysis. The stage of change could be assessed by analyzing the subscale's scores. This study's internal consistency study was 0.794 .

\section{Statistical analysis}

\section{Subscales and reliability}

Factor analysis was performed to determine the subscales of the Internet Addiction Improvement Motivation Scale. It was assumed to consist of three subscales reflecting the first three stages of change according to the theory. Principal axis factor analysis and varimax rotation were conducted by fixing the number of factors at three. Additionally, confirmatory factor analysis was conducted and fit indices are calculated. The internal consistency of each subscale was measured.

\section{Cut-off scores}

To determine cut-off scores to screen for the poorly motivated group using the Internet Addiction Improvement Motivation Scale, descriptive analysis was performed and the scale was standardized.

\section{Validity}

Correlation analysis between the scores of the subscales and the total score of K-SOCRATES-internet was conducted to investigate whether each subscale measured a valid motivation construct.

\section{RESULTS}

\section{Incident rate of internet addiction}

According to K-scale results, $4.4 \%$ of participants appeared to be in the Internet addiction group, and $9.6 \%$ of them appeared to fall into the latent risk group of Internet addition.

\section{Factor structure and internal consistency}

The Internet Addiction Improvement Motivation Scale was found, as predicted when it was composed, to consist of three subscales measuring the motivation levels at pre-contemplation, contemplation, and preparation stages. In this study, the internal consistency recorded $0.613,0.724$, and 0.734 , respectively. The results of the factor analysis and internal consistency of the subscales are shown in Table 2. According

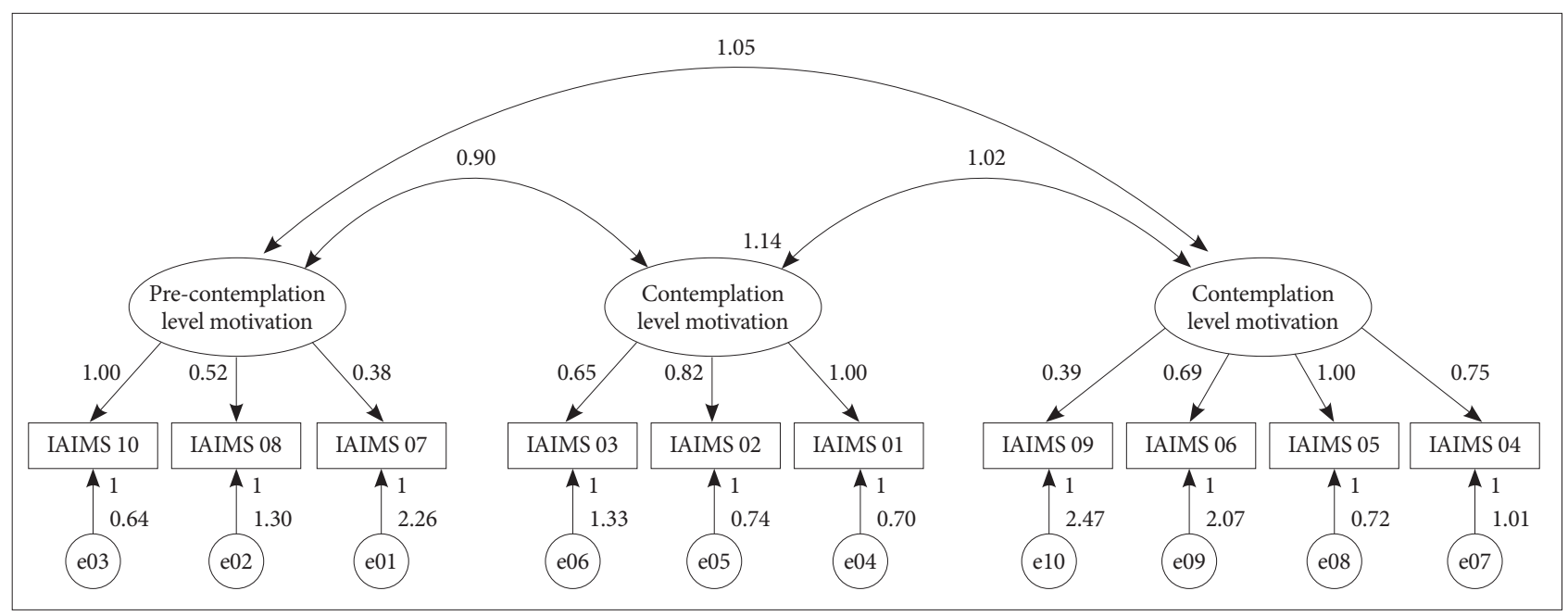

Figure 1. Confirmatory factor analysis results of Internet Addiction Improvement Motivation Scale (IAIMS). 
to the confirmatory factor analysis results, the given factor structure model has shown following level of Fit Indices (GFI= 0.891 , AGFI=0.862, RMSEA=0.089). These results are presented in Figure 1 and Table 3.

\section{Determining cut-off scores to screen for the high-risk group}

To determine cut-off scores to screen for the high-risk group with low motivation to improve Internet addiction, descriptive analysis on the scores of the subscales and the total score was performed. Results are presented in Table 2. According to the results, adolescents recording less than 33 points in the total score or less than 10,11 , or 9 points in the scores of precontemplation, contemplation, or preparation, respectively, were thought to be classified as the high-risk group with low motivation.

\section{Correlation study: validity of the subscales}

The results of the analysis on the correlation of the subscale scores and the total score with K-SOCRATES-I to test the construct validity are shown in Table 4 . According to the data, the scores of contemplation and preparation (except pre-contemplation) and the score of the total scale were significantly related with the total score of K-SOCRATES-I. The correlation coefficients with K-SOCRATES-I were 0.221 ( $\mathrm{p}<0.05) ; 0.340$ $(\mathrm{p}<0.01)$; and $0.341(\mathrm{p}<0.01)$ for contemplation, preparation, and the total scale, respectively. Because the correlation between the subscale of pre-contemplation and K-SOCRATESI was not significant, the subscale could not be considered appropriate as an index to screen for the high-risk group. The subscales of contemplation and preparation and the total scale are considered, however, to be appropriate for indexes.

\section{DISCUSSION}

Many persons with addiction experience various discom-

Table 3. Fit indices

\begin{tabular}{cccc}
\hline & GFI & AGFI & RMSEA \\
\hline Model & 0.891 & 0.862 & 0.089 \\
\hline
\end{tabular}

GFI: Goodness of Fit Index, AGFI: Adjusted Goodness of Fit Index, RMSEA: root mean square error of approximation fort as the addiction becomes more severe, and they are thus motivated to resolve the problem. ${ }^{3}$ In particular, it is common among some clients to seek counseling voluntarily. Counseling to deal with addiction, however, is an area facing clients frequently who are visiting counselors involuntarily. Some of them seek counseling as a part of a legal process, and some adolescents attend counseling involuntarily due to the will of their parents. Internet addiction is more severe in adolescents, with many seeing counselors with insistence from their parents. Some clients without self-motivation cannot be treated effectively through standard counseling because their motivation is very low despite the seriousness of their addiction.

To deal with this problem, this study developed an Internet Addiction Improvement Motivation scale to screen out the poorly motivated group of clients with Internet addiction. The scale was invented by modifying its precursor, the KSCMS, which was developed to measure smoking cessation motivation. The scale was adapted to fit Internet dependence. For addiction, interventions are provided by focusing on the importance of motivation. Motivational enhancement therapy, the effect of which has been proven in many studies, adopts the stage of change model. This change model includes five stages for patients with dependence who wish to recover-precontemplation, contemplation, preparation, action, and maintenance. Because the purpose of the scale was to evaluate the motivation level of clients at the beginning of treatment, the 10 questions reflecting characteristics of thought and behaviors observed in the first three stages-pre-contemplation, contemplation, and preparation-were included. This is because clients in the initial stages of treatment had not yet achieved the action or maintenance stages. Although the KSCMS was found to have the preparation subscale divided into two stages (unlike the theoretical model reflecting the factor structure), this scale specific to Internet addiction was observed to have a three-factor structure, following the theoretical hypothesis very accurately.

The three subscales reflected the motivation levels at precontemplation, contemplation, and preparation stages, and their internal consistency showed acceptable reliability by recording $0.613,0.724$, and 0.734 , respectively. In addition, correlation analysis with K-SOCRATES-I conducted to test the validity revealed that the scores of contemplation and prepar-

Table 4. Correlations of motivation measures

\begin{tabular}{lcccc}
\hline & Precontemplation & Contemplation & Preparation & Motivation-total \\
\hline Contemplation & $0.356^{*}$ & & & \\
Preparation & 0.136 & $0.331^{*}$ & $0.789^{*}$ & $0.341^{* *}$ \\
Motivation-total & $0.642^{*}$ & $0.704^{*}$ & $0.340^{*}$ & $0.221^{*}$ \\
SOCRATES & 0.128 & & & \\
\hline
\end{tabular}

${ }^{*} \mathrm{p}<0.05,{ }^{* *} \mathrm{p}<0.01$ 
ation and the total scale (except pre-contemplation) were significantly related with the total score of the K-SOCRATES-I. The total score and the two subscales were thus found to have acceptable validity.

According to the results of the descriptive analysis to standardize the scale or to determine the cut-off scores for screening the high-risk group, adolescents recording less than 33 points in the total score or less than 11 or 9 points on the subscales of contemplation or preparation, respectively, were classified as the high-risk group with low motivation to be treated for Internet addiction.

Considering the cost efficiency in treating Internet addiction, very intensive motivational enhancement therapy cannot always be provided for all clients. This study developed the Internet Addiction Improvement Motivation scale and showed acceptable reliability and validity. Offering intense interventions to strengthen motivation at the beginning of treatment to the poorly motivated risk group identified with the cut-off scores suggested in this study should increase the success rate and the efficiency of the treatment.

The limitation of this study is that, when it comes to confirmatory factor analysis, the fit indices were not fully acceptable. Although exploratory factor analysis results shows that the K-IAIMS has acceptable factor structure accord with theoretical basis, when the data are fully accumulated confirmatory factor analysis must be performed again.

\section{Acknowledgments}

This study was performed at Hanshin-Pluscare Counseling Center as a part of Community Service Investment Voucher Program of the Korean Ministry of Health \& Welfare and Seoul city. Program Code Number is 4,179 .

\section{REFERENCES}

1. Van Rooij AJ, Schoenmakers TM, Vermulst AA, Van den Eijnden RJ,
Van de Mheen D. Online video game addiction: identification of addicted adolescent gamers. Addiction 2010;106:205-212.

2. Bakken IJ, Wenzel HG, Götestam KG, Johansson A, Oren A. Internet addiction among Norwegian adults: a stratified probability sample study. Scand J Psychol 2009;50:121-127.

3. Cheung LM, Wong WS. The effects of insomnia and internet addiction on depression in Hong Kong Chinese adolescents: an exploratory cross-sectional analysis. J Sleep Res 2011;20:311-317.

4. Goldberg I. Internet Addiction Disorder. Available at: www.psycom. net/iadcriteria.html. Accessed November 20, 2004.

5. Young KS. Psychology of computer use: XL. Addictive use of the Internet: a case that breaks the stereotype. Psychol Rep 1996;79:899-902.

6. Kang HY. An Exploration of Adolescents' Internet Addiction and Game Addiction's Social Self-Regulation Model and Effects of Self-esteem Enhancement Cognitive Behavioral Therapy. Daegu: Kyoung Buk National University; 2009.

7. Kim JS, Choi SM, Kang JS. Computer addiction of Korean adolescents. Seoul: Korea Youth Counseling Institute Press; 2000.

8. Park JW. Internet Addiction and Treatment Motivation, Unpublished Research Article. Catholic University of Korea. 2011, p.3-4.

9. Young KS. Internet Addiction: the Emergence of a New Clinical Disorder. New York: Proceedings of the 104th Annual Conference of the American Psychological Association; 1996.

10. Kim CT, Kim DI, Park JK, Lee SJ. A Study on Internet Addiction Counseling and the Development of Prevention Programs. Seoul: The Korea General Policy Study Project of Information Communication Press; 2002.

11. Miller WR, Rollnick S. Motivational Interviewing: Preparing People to Change Addictive Behavior. New York: Guilford Press; 2002.

12. Park JW, Chai S, Lee JY, Joe KH, Joung EJ, Kim DJ. Validation study of Kim's smoking cessation motivation scale and its predictive implications. Psychiatry Investig 2009;6:272-277.

13. Miller WR, Tonigan JS. Assessing drinkers' motivation for change: the stage of change readiness and treatment eagerness scale (SOCRATES). Psychol Addict Behav 1996;10:81-89.

14. Chun YM. Assessing alcohol dependents' motivation for change: the development study on the Korean version of the stage of change readiness and treatment eagerness scale. Korean J Clin Psychol 2005;24:207-223. 